\title{
A Study of Placental Changes in Polycystic Ovarian Syndrome Patients with Gestational Diabetic Mellitus
}

\author{
Vasantha Lakshmi Ambarapu1, Gunapriya Raghunath², Siva Ranjan D. ${ }^{3}$, Muralidhar Reddy ${ }^{4}$ \\ ${ }^{1}$ Department of Anatomy, Nimra Institute of Medical Sciences, Vijayawada, Andhra Pradesh, India. \\ 2Department of Anatomy, Saveetha Medical College, Kanchipuram, Tamilnadu, India. \\ 3Department of Pathology, Nimra Institute of Medical Sciences, Vijayawada, Andhra Pradesh, India \\ 4Department of Anatomy, NRI Medical Sciences, Guntur, Andhra Pradesh, India.
}

\section{ABSTRACT}

\section{BACKGROUND}

Placenta is a vital organ for the development of foetus and maintenance of pregnancy. Placenta helps in the development and growth of foetus in uterus. In between maternal and foetal circulation the transfer of waste products and nutrients was done by the placenta which is a complex organ of a short life span. The diabetic pregnancy is characterised by numerous disturbances in foetal growth and development.

\section{METHODS}

A cross sectional study on placental changes in 60 pregnant women was done over a period of 18 months. Pregnant women in the age group of 25 years to 40 years who were suffering from polycystic ovarian syndrome (PCOS) were included in the study. Placental specimens were collected from obstetrics department and fixed overnight in $10 \%$ formalin. Thorough gross examination was done and few bits from the abnormal areas were given for microscopic examination. Routine histopathological process was followed for microscopic examination.

\section{RESULTS}

Out of 60 pregnant women with PCOS, seven patients were found to be having PCOS with gestational diabetes mellitus. These seven patients showed gross and microscopic changes in the placenta when compared with non-diabetic PCOS pregnant women.

\section{CONCLUSIONS}

Placenta of diabetic women with PCOS showed both gross and microscopic abnormalities which may affect the growth and development of baby during intrauterine life.

\section{KEY WORDS}

Histopathological Study, Placenta, Polycystic Ovarian Syndrome, Gestational Diabetes
Corresponding Author:

Vasantha Lakshmi Ambarapu,

H. No. C-108, Phase-2, Hill Colony,

Vanasthalipuram, Hyderabad-500070,

Telangana, India.

E-mail: avis.reddy@gmail.com

DOI: 10.14260/jemds/2020/254

Financial or Other Competing Interests: None.

How to Cite This Article:

Ambarapu VL, Raghunath G, Ranjan SD, et al. A study of placental changes in polycystic ovarian syndrome patients with gestational diabetic mellitus. J. Evolution Med. Dent. Sci. 2020;9(14):1171-1174, DOI: $10.14260 /$ jemds/2020/254

Submission 18-12-2019,

Peer Review 13-03-2020

Acceptance 21-03-2020,

Published 06-04-2020.

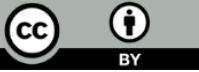




\section{BACKGROUND}

The most common endocrinopathies in the reproductive age group among women is polycystic ovarian syndrome with a prevalence of 7 to $15 \% .^{[1,2]}$ In a few studies, it was described that there will be more metabolic complications seen in nonpregnant women suffering from PCOS, and it was also described that the pregnancy outcomes in women suffering from PCOS remained controversial.[2-4] Gestational diabetes mellitus (GDM) at onset of pregnancy is defined as carbohydrate intolerance which affects $4-7 \%$ of pregnancies.[5] In the reproductive age group of women the most common endocrine disorders are PCOS with the prevalence ranging from $5 \%$ to $15 \%$ and GDM with prevalence ranging from $9 \%$ to $25 \%$. These prevalence of PCOS and Gestational Diabetes Mellitus varies from 5 to $15 \%$ and 9 to $25 \%$ respectively depending on the study group and the criteria applied for diagnosis.[6] The genetic factors, insulin resistance, overweight, metabolic syndrome, dyslipidaemia, cardiovascular risk factors such as hypertension and obesity plays a significant role in PCOS patients associated with gestational diabetic mellitus.[7,8]

Placenta is a vital organ for the development of foetus and maintenance of pregnancy. Placenta helps for the development and growth of foetus in uterus. The present study was done to find out the histopathological changes in placenta and complication seen in PCOS pregnant women with associated GDM and comparison with non-diabetic PCOS pregnant women. In the reproductive health of women over the past few decades in women with PCOS, the pregnancy outcome has received and showed extensive scientific research attention.

In the review of literature over a period of years the data acquired showed many maternal complications. In women with polycystic ovarian syndrome patients, in few metaanalysis studies showed many maternal, neonatal and obstetrics complications and also showed increased risk of other complications like pregnancy induced hypertension, pre-eclampsia, gestational diabetic mellitus and preterm delivery. In the development of these pregnancy complications, placenta plays a major and key role. Polycystic ovarian syndrome is a heterogeneous condition characterized by hyperandrogenism with dysfunction of ovaries. Recent days PCOS is considered as syndrome with metabolic complications affecting health of women at different stages during reproductive and post reproductive life.

The placental morphology with PCOS alone and polycystic ovarian syndrome associated with GDM showed variation both macroscopically and microscopically in many studies. In a particular study the placenta with PCOS alone was examined grossly and microscopically, the gross features was low weight with a reduced volume and smaller in size but microscopically there was no change and there was no complications during pregnancy.[8] In another particular study the placenta with polycystic ovarian syndrome associated with GDM was examined histopathologically, in this study the microscopic changes such as chronic villitis, necrosis was found. However, there was no macroscopic changes and this study also revealed that there was no pregnancy complications in majority of cases and even there was comparison between obese and non-obese women with gestational diabetic mellitus patients.[3]

\section{METHODS}

This is a cross sectional study done on 60 placentas obtained from women suffering from PCOS. The study was done over a period of 18 months. The women of age group of 25 years to 40 years were taken for the study. The sample size was taken based on the convenience of the study. And the placentas obtained from pregnant women suffering from PCOS alone and compared with placentas obtained from women of same age group suffering from PCOS associated with GDM. The clinical data from the patients were obtained from all the 60 patients.

In a teaching hospital, the placentas were obtained from the Obstetrics and Gynaecology department over a period of 18 months and sent to histopathology department for processing. The placentas which were received in the pathology department were fixed overnight in $10 \%$ formalin. The gross examinations of placentas were done on next day. The tissue bits were given for routine histopathology processing from the suspicious and from the abnormal areas of placenta. The tissue bits were passed under graded alcohols in automated tissue processer and different steps of tissue processing to prepare microscopic slides. However the gross examination was also done to find out any abnormalities and microscopic changes in placenta are noted in all the 60 placentas.

\section{Statistical Analysis}

Statistical Analysis was done by using ANOVA for comparison between groups and the $\mathrm{p}$ values $<0.05$ was taken as statistically significant value.

\section{RESULTS}

Out of 60 placentas which were received in the histopathology department, 53 women have only PCOS and seven patients have PCOS associated with GDM. The placental gross features of those seven diabetic PCOS patients are larger in size when compared with the non-diabetic PCOS patients and also a few areas of necrosis. The macroscopic features shown in (Table 1)

\begin{tabular}{|c|c|c|c|c|c|}
\hline \multirow[b]{2}{*}{$\begin{array}{l}\text { Macroscopic } \\
\text { Changes }\end{array}$} & \multicolumn{3}{|c|}{ Mean \pm SD } & \multirow[b]{2}{*}{$\begin{array}{c}\text { p } \\
\text { Value }\end{array}$} & \multirow[b]{2}{*}{ Significance } \\
\hline & Controls & PCOS & $\begin{array}{l}\text { PCOS with } \\
\text { GDM }\end{array}$ & & \\
\hline $\begin{array}{l}\text { Placental diameter } \\
\text { in centimetres }\end{array}$ & $16.2-+2.05$ & $16.7-+3.06$ & $16.10-+2.53$ & $>0.005$ & not significant \\
\hline $\begin{array}{l}\text { Placental Thickness } \\
\text { in cms }\end{array}$ & $2.6-+0.43$ & $2.5-+0.42$ & $2.6-+0.45$ & $>0.005$ & not significant \\
\hline $\begin{array}{l}\text { Placental weight } \\
\text { in grams }\end{array}$ & $450-+73.8$ & $410-+15.3$ & $413-+47.3$ & $<0.03$ & significant \\
\hline $\begin{array}{l}\text { Number of } \\
\text { cotyledons in } \\
\text { placenta }\end{array}$ & $18.3-+1.47$ & $17.5-+1.28$ & $17.6-+1.40$ & $>0.005$ & not significant \\
\hline $\begin{array}{l}\text { Baby weight in } \\
\text { grams }\end{array}$ & $3.10-+0.16$ & $2.10-+0.24$ & $2.65-+0.24$ & $<0.01$ & significant \\
\hline $\begin{array}{l}\text { Table } 1 \\
\text { Compa }\end{array}$ & . & 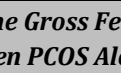 & 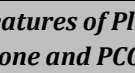 & I & $\begin{array}{l}\text { and } \\
\text { anM }\end{array}$ \\
\hline
\end{tabular}


The microscopic changes are shown in (Table 2) which was seen in Diabetic PCOS placenta and non-diabetic PCOS placentas. Only 16 placentas out of 53 non-diabetic PCOS placentas showed the microscopic changes but the entire seven diabetic PCOS placenta showed histopathological changes.

\begin{tabular}{|ccc|}
\hline Microscopic & Diabetic PCOS & Non-Diabetic PCOS \\
Features & Placenta $(\mathbf{n}=7)$ & Placenta $(\mathbf{n = 5 3})$ \\
Villous edema & 7 & 2 \\
Fibrinoid necrosis & 7 & 9 \\
Dilated blood vessels & 7 & 5 \\
\hline \multicolumn{2}{|c|}{ Table 2. Showing the Microscopic Changes in Placentas } \\
\hline
\end{tabular}

\section{DISCUSSION}

Placenta is a vital organ for maintenance of pregnancy and for the development and growth of fetus in uterus. PCOS is a heterogenous condition characterized by hyperandrogenism with dysfunction of ovaries. Women suffering from PCOS are more prone for developing diabetes mellitus during pregnancy; similarly, women who are diagnosed as GDM are most likely to have underlying polycystic ovarian disease.[9,10] In our study out of 60 patients we found seven (11.67\%) gestational diabetic mellitus patients associated with PCOS. The insulin status during pregnancy is regulated by placental progesterone, estradiol, cortisol and lactogen. During the third trimester of pregnancy the physiological insulin resistant state reaches its peak, so the transfer of glucose level to fetus and due to the facilitated diffusion the glucose levels are maintained.[9] The pregnancy complications which may arise in women who is suffering from PCOS are due to pre-existing insulin resistant state.

In review of literature the method to investigate the association of diabetes mellitus during pregnancy with PCOS they used multivariable logistic regression. They found that the women with higher maternal age, multiple gestation, Hispanic ethnicity and Asian race were more prone for increased risk of diabetes in associated PCOS pregnant women. ${ }^{[11]}$ The present study also showed that all the diabetic PCOS patients were of high maternal age when compared with non-diabetic patients. Similarly a few other studies showed that there is an increase of risk for the development of diabetic mellitus in women suffering from PCOS during pregnancy when compared to the normal pregnant women and even elderly women, multiple gestation, Hispanic ethnicity and Asian race were of more prone for developing diabetic mellitus.

Among different studies in literature metformin therapy showed different results. Zeng XL et al in their study reported the effects of metformin therapy throughout pregnancy lowered the risk of diabetic mellitus in PCOS patients. [12-14] Meta-analysis by other three studies concluded that metformin therapy has no significance among PCOS patients associated with GDM.[15,16] In our present study the clinical history was obtained from all the patients; but none of the patients have used metformin therapy. However, in the review of literature showed that the usages of metformin before conception and throughout the pregnancy are inconclusive and prevention of diabetic mellitus in PCOS are still controversial and inconclusive.
In present study the placentas received from obstetrics and gynaecology department were fixed overnight in $10 \%$ formalin in histopathology department. The gross examination of these placentas were done, majority of the placentas are in normal size and shape with weight varying from 400 to 550 grams. Two placentas showed umbilical cord insertion at eccentric, but the fetal growth was normal and no complications found in the fetus. The other gross features observed in this study was placentas are irregular in shape and a few placentas showed dilated umbilical cord. A part from these gross features one of the placenta showed less cotyledons and these cotyledons were also not prominent. However, there were no signs of vascular damage followed by fetal hypoxia.

The placentas obtained from women with PCOS associated with GDM have different morphological feature, both gross and microscopically from placentas obtained from women with PCOS alone. Review of literature, one of the study showed the comparison between placentas obtained from women with PCOS and placentas from the normal pregnant women, In this study it was discussed that the placentas obtained from PCOS patients showed few gross changes like less in weight, reduced volume and thin when compared to the placentas obtained from normal healthy pregnant women. In this study microscopically it has been observed that placentas obtained from women with PCOS have more villous oedema and intervillositis. However, to discuss that this study included only a few selected group of women with PCOS and who did not developed any pregnancy complications and who were non-obese and conceived spontaneously, so therefore this study will not represent the overall spectrum of the heterogeneous PCOS population. [3] In present study, all the placentas received from gestational diabetic mellitus patients were larger in size and heavy when we compared it to other placentas of non-diabetic PCOS patients. The histopathological microscopic examination of diabetic women placenta showed numerous pathological abnormalities such as villous oedema, fibrinoid necrosis, and dilated blood vessels. Similar microscopic changes were seen in other studies.[7]

Review of literature showed that there are several maternal, neonatal obstetric complications in polycystic ovary syndrome patients. Multiple gestations are most important cause for the morbidity during perinatal period. Even in review of literature a few Meta-analysis study showed that there were increased risk of pregnancy complications in women suffering from PCOS. Regarding miscarriages it is still not yet concluded when the women has Polycystic ovaries. In our present study we did not found any major complications related to neonate are maternal a part from two neonates who were born with low birth weight. In PCOS patients who were conceived will have more risk for developing diabetic mellitus as pregnancy complications, in such cases early diagnosis and screening will reduce the incidence of diabetic mellitus and also prevents the complications related to mother and fetus.

A few studies described about the maternal age, maternal weight and polycystic ovaries are related to the development of diabetic mellitus during pregnancy. For these women who has a risk for developing diabetes mellitus should be recognized earlier before conception and counseling should be given to achieve therapeutic interventions more efficiently 
and also to prevent complications during pregnancy and even after pregnancy.[16] In our present study there are seven gestational diabetes women associated with PCOS, out of seven GDM patients three patients are high maternal age over 36 years and among these three patients all of them are overweight. However, all the seven GDM patients are overweight exceeding 85 kilograms but none of them have a family history of diabetes mellitus.

\section{CONCLUSIONS}

Placenta of diabetic women with PCOS showed both gross and microscopic abnormalities which may affect the growth and development of a baby during intrauterine life. However, no major complications were seen in those seven diabetic PCOS pregnant women.

\section{REFERENCES}

[1] Tehrani FR, Simbar M, Tohidi M, et al. The prevalence of polycystic ovary syndrome in a community sample of Iranian population: Iranian PCOS prevalence study. Reprod Biol Endocrinol 2011;9:39.

[2] Dennett CC, Simon J. The role of polycystic ovary syndrome in reproductive and metabolic health: overview and approaches for treatment. Diabetes Spectr 2015;28(2):116-20.

[3] Palomba S, Santagni S, Falbo A, et al. Complications and challenges associated with polycystic ovary syndrome: current perspectives. Int J Womens Health 2015;7:74563.

[4] Teede H, Deeks A, Moran L. Polycystic ovary syndrome: a complex condition with psychological, reproductive and metabolic manifestations that impacts on health across the lifespan. BMC Med 2010;8:41.

[5] Ferrara A, Kahn HS, Quesenberry CP, et al. An increase in the incidence of gestational diabetes mellitus: Northern
California, 1991-2000. Obstet Gynecol 2004;103(3):52633.

[6] Norman RJ, Dewailly D, Legro RS, et al. Polycystic ovary syndrome. Lancet 2007;370(9588):685-97.

[7] Bhathena RK. Insulin resistance and the long-term consequences of polycystic ovary syndrome. Journal of Obstetrics and Gynaecology 2011;31(2):105-10.

[8] Goueslard K, Cottenet J, Mariet AS, et al. Early cardiovascular events in women with a history of gestational diabetes mellitus. Cardiovascular Diabetology 2016;15:15.

[9] Mikola M, Hiilesmaa V, Halttunen M, et al. Obstetric outcome in women with polycystic ovarian syndrome. Hum Reprod 2001;16(2):226-9.

[10] Anttila L, Karjala K, Penttila RA, et al. Polycystic ovaries in women with gestational diabetes. Obstet Gynecol 1998;92(1):13-6.

[11] Franks S. Polycystic ovary syndrome. N Engl J Med 1995;333(13):853-61.

[12] Zeng XL, Zhang YF, Tian Q, et al. Effects of metformin on pregnancy outcomes in women with polycystic ovary syndrome: a meta-analysis. Medicine (Baltimore) 2016;95(36):e4526.

[13] Zhuo Z, Wang A, Yu H. Effect of metformin intervention during pregnancy on the gestational diabetes mellitus in women with polycystic ovary syndrome: a systematic review and meta-analysis. J Diabetes Res 2014;2014:381231.

[14] Zhao J, Liu X, Zhang W. The effect of metformin therapy for preventing gestational diabetes mellitus in women with polycystic ovary syndrome: a meta-analysis. Exp Clin Endocrinol Diabetes 2020;128(3):199-205.

[15] Feng L, Lin XF, Wan ZH, et al. Efficacy of metformin on pregnancy complications in women with polycystic ovary syndrome: a meta-analysis. Gynecol Endocrinol 2015;31(11):833-9.

[16] Tan X, Li S, Chang Y, et al. Effect of metformin treatment during pregnancy on women with PCOS: a systematic review and meta-analysis. Clin Invest Med 2016;39(4):E120-31. 